MIDPI

MOL2NET, International Conference Series on Multidisciplinary Sciences http://sciforum.net/conference/mol2net-03

sciforum

\title{
Exploring different strategies to improve the magnetic response of cobalt doped ferrite nanoparticles
}

Itziar Galarreta (itziar.galarreta@ehu.eus) ${ }^{a}$, Maite Insausti (maite.insausti@ehu.eus) ${ }^{a}$,b, Izaskun Gil de Muro (izaskun.gildemuro@ehu.eus) ${ }^{\text {a,b }}$, Idoia Ruiz de Larramendi (idoia.ruizdelarramendi@ehu.eus) ${ }^{\text {a }}$, Luis Lezama (luis.lezama@ehu.eus) ${ }^{\text {a,b }}$.

${ }^{a}$ Dpto. de Química Inorgánica, Universidad Del País Vasco, UPV/EHU, P.O. Box. 644, E-48080 Bilbao, Spain.

${ }^{b}$ BCMaterials, Parque Científico y Tecnológico de Bizkaia, E-48160 Derio, Spain.

\section{Graphical Abstract}

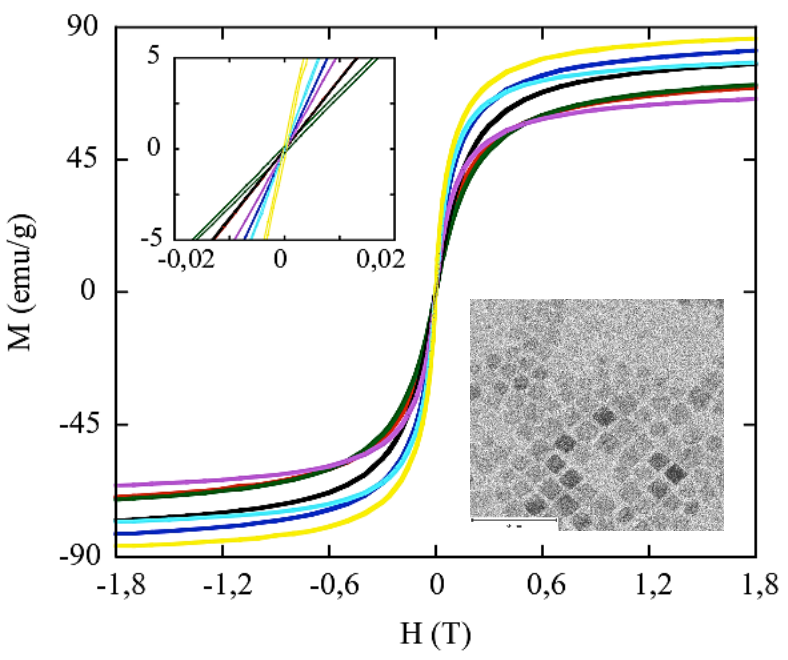

\begin{abstract}
.
With the aim of studying the structural and magnetic properties of cobalt doped magnetite nanoparticles $\left(\mathrm{Fe}_{3-x} \mathrm{Co}_{x} \mathrm{O} 4\right)$, several samples were synthetized by thermal decomposition method using different cobalt concentrations $(0-5 \%)$ at different condition reflux time $(30$ - $120 \mathrm{~min})$. In this work, we demonstrate that the synthetic parameters highly influence. Both, the morphology and the cobalt concentration, obtaining higher saturation magnetization values for 2 hours reflux. An exhaustive magnetic characterization by means of magnetization and electronic magnetic resonance has established conditions to improve the magnetic response of doped nanoparticles.
\end{abstract}

\section{Introduction (optional)}

The application of magnetic nanoparticles in therapies based on localized magnetic hyperthermia depends directly on their size, composition and biocompatibility. Hyperthermia therapy is based on the fact that tumor cells are more thermosensitive than healthy cells, so that raising the temperature to a certain value could selectively damage the cancer cells [1]. They must present sufficient size to generate the necessary heat in the localized area and produce the necrosis always maintaining its superparamagnetic character. Certainly, the magnetic saturation derived from the composition of the material will be primarily responsible for the generation of heat in magnetic hyperthermia, being one of the key factor that defines the possible efficacy of such technique [2].

With the aim of studying the structural and magnetic properties of cobalt doped magnetite nanoparticles $\left(\mathrm{Fe}_{3-\mathrm{x}} \mathrm{Co}_{\mathrm{x}} \mathrm{O}_{4}\right)$, different cobalt concentrations $(0-5 \%)$ and different synthesis conditions have been employed to prepare the nanostructures. Nanoparticles have been structural and morphological characterized and magnetic properties have been studied by measuring magnetization at different temperatures and applied fields. Electron Magnetic Resonance (EMR) measurements have been carried out as this technique can provide useful information on particle evaluation, shape and surface effects or inter-particle interactions. 


\section{Materials and Methods}

Several samples were synthetized by thermal decomposition of iron (III) acetylacetonate and cobalt (II) acetylacetonate in a solvent of a high boiling point and in the presence of oleic acid, oleylamine and 1, 2-hexadecanediol [3 - 4]. The mixture was first heated at $200{ }^{\circ} \mathrm{C}$ for $30 \mathrm{~min}$ (nucleation phase) and then was ramped with a heating rate of about $2 \%$ min to the reaction temperature of $295^{\mathrm{a}} \mathrm{C}$ in $\operatorname{argon}$ atmosphere. Synthesis was performed at various times to investigate the morphological evolution of the NPs. After the solution was cooled to room temperature, the resulting nanoparticles were precipitated with ethanol and magnetic separated using a magnet.

\section{Results and Discussion}

The structural characterization of samples was made by X-ray diffraction (XRD). The intensity and peak positions of the diffraction patterns are in good agreement with face-centred cubic phase of magnetite (Fd-3m, JCPDS No. 89-0691). No traces of any kind of detectable impurities were observed. The main effect of changing the reflux times is related to the amount of cobalt introduced in the ferrite lattice, as confirmed by ICP analysis. Longer reaction times give rise to an increase in the effectiveness of doping due to the higher temperatures needed for the decomposition of the Co-precursor in comparison with the $\mathrm{Fe}$ one. Mass evolution observed in thermogravimetric analysis under Ar flow has been similar for all samples. The first great mass loss (of around 10\%) occurs between 300 and $420^{\circ}$ $\mathrm{C}$, which can be associated to the decomposition of weakly attached surface functional coating or capping groups. The second mass loss of about $20 \%$, between 550 and $650^{\circ} \mathrm{C}$ is related with the final decomposition of ligands surrounding the magnetic core. The total amount of organic matter is between $28-44 \%$. TEM micrographs of all samples show well-dispersed homogeneous nanoparticles with sizes between 6 and $11 \mathrm{~nm}$ and morphologies depending on the reaction times, varying from spheric, cuboctahedral to cubic.

Magnetic studies have been performed by macroscopic magnetometry at different temperatures and fields. Measurements of magnetization versus temperature after Cooling at Zero Field (ZFC) and Field (FC) show the usual characteristics of a superparamagnetic (SPM) behaviour. The substituted samples blocking temperatures, $\mathrm{T}_{\mathrm{B}}$, vary in the $46-110 \mathrm{~K}$ interval depending on size, anisotropy constant and Co content, in good accord with the Stoner-Wohlfarth theory [5]. The SPM character of samples has been additionally confirmed by the absence of coercive field $\left(\mathrm{H}_{\mathrm{C}}\right)$ in the hysteresis loops recorded at R.T. Magnetization values obtained at R.T. vary between 65 and $86 \mathrm{emu.g}^{-1}$, corresponding the maximum values to samples with the highest Co contents and with the highest times of reflux temperatures. Nevertheless, variations of the saturation values from one sample to another has been analyzed and interpreted taking into account the modulation of antiferromagnetic interactions between $\mathrm{T}_{\mathrm{d}}$ and $\mathrm{O}_{\mathrm{h}}$ sites occupied by magnetic cations. EMR spectra recorded at R.T in colloidal medium complete the information concerning the magnetic characteristics of the different samples. The spectra exhibit anisotropic lines different than the previously observed nearly Gaussian lines, characteristic of magnetite nanoparticles [3]. This fact corroborates the formation of cobalt doped ferrite nanoparticles.

\section{Conclusions}

This work provides a deeper insight into the obtaining of cobalt doped ferrite nanoparticles with $\mathrm{Fe}_{3}$ ${ }_{x} \mathrm{Co}_{\mathrm{x}} \mathrm{O}_{4}$ general formula. The ability to tune the amount of cobalt in the ferrite lattice is due to variations of reaction times during the synthesis. These changes do not seem to affect significantly the average particle size or the size distribution. Magnetic data confirm the dependence of blocking temperatures with the cobalt content. Thus, higher magnetizations are related to longer reflux time and, consequently, with higher amounts of cobalt in the ferrite nanoparticles.

\section{References}

[1] Marszall, M. P. Pharm. Res., 2001, 28 (3), 1173.

[2] Lartigue, L. et al, J. of Am. Chem. Soc., 2011, 133, 10459.

[3] Castellanos-Rubio, I. et al, Nanoscale, 2014, 6(13), 7542

[4] Lasheras X. et al., J. Phys. Chem. C, 2016, 120, 3492.

[5] Stoner, E.C. and Wohlfarth, E.P. Phios. Trans. R. Soc. London, Ser. A 1948, 240, 599. 